\title{
CONVOLUTION MEASURE ALGEBRAS WITH INVOLUTION
}

\author{
J. W. BAKER
}

ABSTRACr. Let $S$ be a commutative, locally compact semigroup and $A$ an $L$-subalgebra of $M(S)$. We obtain conditions on $A$ to ensure that $S$ is an inverse semigroup, a group, or an idempotent semigroup. We assume an involution on $A$, or equivalently on $S$. The proofs come readily from known facts.

Let $S$ be a locally compact, jointly continuous, commutative, topological semigroup, and let $M(S)$ denote the algebra of bounded, regular Borel measures on $S$. Suppose that $A$ is a complex, $L$-subalgebra of $M(S)$ (see [1]). We wish to investigate the problem of finding conditions on $A$ to ensure that $S$ belongs to certain classes of topological semigroups. We shall consider three classes of topological semigroups: (a) the inverse semigroups; (b) groups; and (c) idempotent semigroups. We shall regard (b) and (c) as being two subclasses of (a). One particular situation in which the problem has been studied is that in which $S$ is taken to be the 'structure semigroup' of $A$ (see [1]). In that case, quite a lot is already known; (a) is studied in [1], (b) in [2] and [3], and (c) in [4]. We hope that what follows will serve to unify the results obtained in the above-mentioned papers, and at the same time provide an extension to other important casesfor example, when $A$ is $l^{1}(S)$. We also feel that our solution to (b) has certain advantages over those in [2] and [3] in its simplicity.

It is clearly necessary to make some additional assumptions about the relationship between $A$ and $S$ in order to be able to deduce information about $S$ from knowledge of $A$. Let $A^{\wedge}$ denote the space of nonzero, complex homomorphisms of $A$, and $S^{\wedge}$ the set of semicharacters of $S$ (nonzero, continuous homomorphisms of $S$ into the unit disc). We must first assume that

(A) the carrier space of $A$ is the whole of $S$.

This assumption is equivalent to assuming that $A$ is $\sigma\left(M(S), C_{0}(S)\right)$-dense in $M(S)$. If (A) is satisfied then there is a natural mapping $\eta$ of $S^{\wedge}$ into $A^{\wedge}$ defined by the equation

Received by the editors May 12, 1970.

AMS 1970 subject classifications. Primary 43A10; Secondary 20M30, 22A15, $43 \mathrm{~A} 05,43 \mathrm{~A} 40$.

Key words and phrases. Banach algebras, convolution measure algebras, locally compact semigroups, structure semigroups.

Copyright (c) 1971, American Mathematical Society 


$$
(\eta(\chi))(\mu)=\int \chi(x) d \mu(x) \quad(\mu \in A, \chi \in \hat{S}) .
$$

We shall also assume that

(B) $\eta$ defines a (set-theoretical) one-one correspondence between $S^{\wedge}$ and $A^{\wedge}$.

Finally, we shall assume that

(C) A is semisimple, and $S^{\wedge}$ separates the points of $S$.

The two assumptions contained in (C) are closely related, but we have been unable to show that they are always equivalent. Our assumptions (A), (B), and (C) are certainly true if $S$ is discrete and $A$ is $l^{1}(S)$-provided that $A$ is semisimple (see [5])-or if $S$ is the structure semigroup of the semisimple algebra $A$.

For the rest of this paper we shall assume that $A$ is an $L$-subalgebra of $M(S)$ for which (A), (B), and (C) are true. Problem (a) is that of finding out when it is true that $S$ is an inverse semigroup. To be more precise, we shall try to find out when $S$ is a continuous-inverse semigroup, that is, a toplogical semigroup and inverse semigroup in which the operation of inversion is continuous. Every compact or discrete inverse semigroup is a continuous-inverse semigroup (see [6]), but this is not true for locally compact inverse semigroups. A continuousinverse semigroup becomes a topological semigroup with continuous involution (in the sense of [7]) if one takes the involution to be the operation of inversion. Suppose that $S$ is a locally compact, topological semigroup with a continuous involution *. Then $C_{0}(S)$ and $M(S)$ will each in turn inherit mappings * defined by the equations

$$
f^{*}(x)=\left(f\left(x^{*}\right)\right)^{-}, \mu^{*}(f)=\left(\mu\left(f^{*}\right)\right)^{-} \quad\left(x \in S, f \in C_{0}(S), \mu \in M(S)\right) .
$$

Clearly $*$ is a positive $\left(\mu^{*} \geqq 0\right.$ if $\left.\mu \geqq 0\right)$, isometric, $\sigma\left(M(S), C_{0}(S)\right)$ continuous, algebra involution on $M(S)$. As in [1] (Theorem 3.4), we shall investigate conditions on a *-subalgebra of $M(S)$, where $S$ is a semigroup with continuous involution, which ensure that $S$ is of type (a), (b), or (c) with the involution as the inverse semigroup inversion.

Observe that if $A$ is given a positive, isometric, $\sigma\left(M(S), C_{0}(S)\right)$ continuous, algebra involution $*$ then $S$ will inherit an involution $*$ for which equations (2) are valid. Further, if $S$ is compact then the $\sigma\left(M(S), C_{0}(S)\right)$-continuity of $*$ is a consequence of its isometry. (In particular, this means that if $A$ is an abstract convolution measure algebra, and $S$ is the structure semigroup of $A$, then $S$ becomes a semigroup with involution $*$ for which equations (2) are valid.) Thus our assumption that $S$ has a continuous involution and $A$ is a *-subalgebra of $M(S)$ is equivalent to the assumption that $A$ has a positive, isometric, $\sigma\left(M(S), C_{0}(S)\right)$-continuous involution. Finally, it is clear 
from Theorem 3.4 of [1] that if $A$ is a symmetric algebra then the involution on $A$ is automatically both positive and isometric. It would seem reasonable to expect to be able to prove this directly, but we have been unable to do so.

Proposition 1. (Cf [1, Theorem 3.4].) Let $S$ be a locally compact, commutative, topological semigroup, with continuous involution *. Suppose that $A$ is an L-subalgebra of $M(S)$, closed under *, with properties (A), (B), and (C). Then $S$ is an inverse semigroup, with * as inversion, if and only if $A$ is symmetric.

Proof. Theorem 3.4 of [7] tells us that $S$ is an inverse semigroup, with involution as its inversion, if and only if $\chi\left(x^{*}\right)=(\chi(x))$ - for every $\chi$ in $S^{\wedge}$ and every $x$ in $S$. This is so if and only if $\int \chi\left(x^{*}\right) d \mu(x)$ $=\int(\chi(x))-d \mu(x)$ for every $\chi$ in $S^{\wedge}$ and every $\mu$ in $M(S)$. Since $A$ is dense in $M(S)$, the latter is true if and only if

$$
\int \chi\left(x^{*}\right) d \mu(x)=\int(\chi(x))-d \mu(x)
$$

for every $\chi$ in $S^{\wedge}$ and every $\mu$ in $A$. So $S$ is an inverse semigroup if and only if

$$
\int \chi(x) d \mu^{*}(x)=\left(\int \chi(x) d \mu(x)\right)^{-}
$$

for every $\chi$ in $S^{\wedge}$ and every $\mu$ in $A$, that is if $h\left(\mu^{*}\right)=(h(\mu))$ - for every $h$ in $A^{\wedge}$ and every $\mu$ in $A$.

Proposition 2. Let $A$ and $S$ be as in Proposition 1. Then $S$ is a group, with * as its inversion, if and only if (i) $A$ is symmetric, and (ii) there is only one positive linear functional $h$ in $A^{\wedge}$.

Note. The unique positive linear functional $h$ in $A^{\wedge}$ will be the functional which satisfies the equation $h(\mu)=\|\mu\|$ for $\mu \geqq 0$.

Proof. Condition (i) is precisely what is required to make $S$ into an inverse semigroup. Given that $S$ is an inverse semigroup, $S$ will be a group if and only if $S^{\wedge}$ is a group, that is if and only if $S^{\wedge}$ has exactly one idempotent. The idempotent semicharacters of an inverse semigroup are precisely those that are real and positive. So, under condition (i), it is clear that $S$ is a group if and only if $S^{\wedge}$ has only one real and positive semicharacter, which is precisely what is ensured by condition (ii).

Corollary. A semisimple convolution measure algebra $A$ with involution is isometrically *-isomorphic to $L^{1}(G)$ for some locally compact group $G$ if and only if it satisfies conditions (i) and (ii) and is Tauberian (that is, salisfies some form of Wiener's Tauberian Theorem; for example, every closed ideal is contained in a maximal regular ideal). 
Proof. It is shown in [2] that if $A$ is a semisimple convolution measure algebra, and is Tauberian, then $A$ is isomorphic to $L^{1}(G)$ if and only if its structure semigroup is a compact group, which turns out to be the Bohr Compactification $\beta(G)$ of $G$ (see [2, Theorem 2]). Now $L^{1}(G)$ is a semisimple, commutative measure algebra with involution, and the involutions on $L^{1}(G), G$, and $\beta(G)$ are clearly compatible in the sense of equations (2). If we apply Proposition 2 to $A$, taking $S$ to be the structure semigroup of $A$, we see that $S$ is a group if and only if $A$ has properties (i) and (ii) and is Tauberian. Observe that, as remarked in the paragraph immediately preceding Proposition 1 , it is not necessary to assume that the involution on $A$ is positive or isometric, in view of Theorem 3.4 of [1].

Proposition 3. (Cf. [4, Theorem 1].) Let $S$ be a locally compact, commutative, topological semigroup, and $A$ an L-subalgebra of $M(S)$, with properties (A), (B), and (C). Then $S$ is an idempotent semigroup if and only if every $h$ in $A^{\wedge}$ is a positive linear functional.

Proof. This proposition is proved in [4], but we offer a proof here based on the ideas of our earlier proofs. The idea of an involution on $S$ and on $A$ is still implicit in the above theorem; the natural involution on an idempotent semigroup is the identity map, which will induce the involution of complex conjugation onto its measure algebra, so that these involutions do not have to be explicitly assumed. In view of Corollary 3.8 of [7], $S$ is idempotent if and only if every element of $S^{\wedge}$ is real and positive. The proof is now clear if one argues as in the proof of Proposition 1.

The following proposition follows by a similar application of Corollary 3.7 of [7].

Proposition 4. Let $A$ and $S$ be as in Proposition 3. In order that every $h$ in $A^{\wedge}$ be real, it is necessary and sufficient that $x^{2}$ be idempotent for each $x$ in $S$.

Proposition 2 tells us that if we wish the structure semigroup of a semisimple, convolution measure algebra $A$ to be a group $G$, with $A$ embedded as a $*$-subalgebra of $M(G)$, it is necessary and sufficient that (i) and (ii) should be satisfied. In general, it is possible to have semisimple, convolution measure algebras whose structure semigroup is a group $G$ but which are naturally embedded in $M(G)$ in such a way that they do not form *-subalgebras of $M(G)$ (see [2]). However, Taylor [2, Theorem 2], has shown that if the structure semigroup of $A$ is a group then there exists a locally compact group $G$ for which $A$ is embeddable in $M(G)$ so that (a) $L^{1}(G) \subseteq A$, and (b) the Jacobson 
Radical of $L^{1}(G)$ in $A$ is the whole of $A$ (otherwise $A \subseteq L^{1 / 2}(G)$ ). Thus, if we think of (b) as saying that $L^{1}(G)$ is a 'large' subset of $A$, then (a) and (b) together say that $A$ contains a large subalgebra which satisfies (i) and (ii).

In the following theorem we use the convention that if $Y$ is a subspace of the normed vector space $X$, and $f$ is in $X^{\prime}$, then $\|f\|_{Y}$ denotes the norm of $f$ as a bounded linear functional on $Y$.

Theorem 5. Let $A$ be a semisimple, convolution measure algebra. $A$ necessary and sufficient condition for the structure semigroup of $A$ to be $a$ group is that,

( $\alpha$ ) A contains an L-subalgebra $B$ which is symmetric,

( $\beta)$ for each finite linear combination $\sum \lambda_{i} h_{i}$ of elements of $A^{\wedge}$, $\left\|\sum \lambda_{i} h_{i}\right\|_{A}=\left\|\sum \lambda_{i} h_{i}\right\|_{B}$, and

$(\gamma)$ there is exactly one positive linear functional in $B^{\wedge}$.

Proof. The necessity of the conditions is simple to establish. If we think of $A$ as being embedded in $M(G)$, with $L^{1}(G) \subseteq A \subseteq L^{1 / 2}(G)$, then we take $B$ to be $L^{1}(G)$. Now $B$ will certainly be symmetric, and will have property $(\gamma)$ (for example, this follows from Proposition 2). A finite linear combination $\sum \lambda_{i} h_{i}$ of elements of $A^{\wedge}$ can be identified with a function $f$ in $C(G)$. Then

$$
\|f\|_{L^{1}}=\sup _{x \in G}|f(x)|=\|f\|_{M(G)}, \quad \text { so that }\|f\|_{B}=\|f\|_{A} \text {; }
$$

this establishes $(\beta)$.

Suppose that the conditions are satisfied. Let $S$ be the structure semigroup of $A$, so that $C(S)$ can be identified with the closed linear span of $A^{\wedge}$ in $A^{\prime}$ (see [1]). In view of condition $(\beta)$, it is clear that the closure can be taken in the norm $\|\cdot\|_{B}$. It follows that $B$ is $\sigma(M(S), C(S)$ )-dense in $A$-and so in $M(S)$. Applying Proposition 2, bearing in mind our remarks immediately preceding Proposition 1 , we are able to conclude that $S$ is a group.

Note. It is easy to determine which subset of $A$ is to play the role of $B$ in the above theorem, so that one just has to check that this set has properties $(\alpha),(\beta)$, and $(\gamma)$. On one hand $B$ will be the smallest ideal of $A$ which satisfies Wiener's Tauberian Theorem, and has the same maximal ideal space as $A$; alternatively, $B$ is the largest symmetric, $L$-subalgebra of $A$.

\section{REFERENCES}

1. J. L. Taylor, The structure of convolution measure algebras, Trans. Amer. Math. Soc. 119 (1965), 150-166. MR 32 \#2932. 
2. - L-subalgebras of $M(G)$, Trans. Amer. Math. Soc. 135 (1969), 105-113. MR $38 \# 1472$.

3. M. A. Rieffel, $A$ characterisation of commutative group algebras and measure algebras, Trans. Amer. Math. Soc. 116 (1965), 32-65. MR 33 \#6300.

4. S. E. Newmann, Measure algebras and idempotent semigroups, Pacific J. Math. 31 (1969), 161-169.

5. E. Hewitt and H. S. Zuckerman, The $l_{1}$-algebra of a commutative semigroup, Trans. Amer. Math. Soc. 83 (1956), 70-97. MR 18, 465.

6. A. D. Wallace, The structure of topological semigroups, Bull. Amer. Math. Soc. 61 (1955), 95-112. MR 16, 796.

7. Anne C. Baker and J. W. Baker, Duality of topological semigroups with involution, J. London Math. Soc. 44 (1969), 251-260. MR 38 \#4596.

The University of Sheffield, Sheffield, Great Britain 